\title{
Platelet peroxidase deficiency in a case of myelodysplastic syndrome with myelofibrosis
}

\author{
MICHËLE IMBERT, MARIE THÉĖSE JARRY, MICHEL TULLIEZ, JEANINE \\ BRETON-GORIUS
}

From the Service Central d'Hématologie-Immunologie et Unité de Recherche sur les anémies, Inserm U91, Hôpital Henri Mondor Créteil, France

SUMMARY Morphological and functional abnormalities of the megakaryocytic series have been well described in myelodysplastic syndromes. Platelet peroxidase has always been demonstrated in abnormal megakaryocytes and early megakaryoblasts in such syndromes. We have studied a case of myelodysplastic syndrome with marked morphological abnormalities of megakaryocytes in which ultrastructural studies showed the coexistence of platelet peroxidase positive and platelet peroxidase negative megakaryocytes. This enzymatic deficiency was confirmed by the ultrastructural study of circulating platelets. This case appears to be the first report of a partial platelet peroxidase deficiency. It adds to the enzymatic abnormalities in myelodysplastic syndrome already described for the red cells and the granulocytic cells.

Myelodysplastic syndromes are considered as myeloid stem cell disorders ${ }^{12}$ characterised by qualitative defects in one or more cell lines accounting for the ineffective myelopoiesis. Besides the morphological abnormalities, numerous other defects have been described in the erythroblastic series-for example, abnormalities of red cell enzymes, ${ }^{3}$ blood group antigens, ${ }^{4}$ possible presence of paroxysmal nocturnal haemoglobinuria clone. ${ }^{5}$ Defects of the granulocytic series have also been recognised, especially anomalies of the granules. They may be quantitative (hypogranularity), qualitative (myeloperoxidase deficiency), functional (abnormal phagocytosis and/or bactericidal activity). ${ }^{6}$ In these cases various subpopulations of normal and abnormal cells coexist. Abnormalities of the megakaryocytic series have also been reported. ${ }^{\circ}$

We describe here a case in which besides the dyserythropoiesis and dysgranulopoiesis, the megakaryocytes showed unusual morphological abnormalities and hitherto undescribed enzymatic deficiency.

\section{Material and methods}

\section{CASE REPORT}

A 70-year-old patient was referred in October 1980 for a problem of macrocytic anaemia. Physical

Accepted for publication 12 July 1983 examination on admission revealed no organomegaly. Haematological findings were $\mathrm{Hb} 8.5 \mathrm{~g} / \mathrm{dl}$; MCV $107 \mathrm{fl}$; MCH $35.4 \mathrm{pg}$; reticulocyte count $51 \times 10^{\%} / \mathrm{l}$; platelets $53 \times 10^{9} / 1$; WBC $9.1 \times 10^{9} / 1$; with $75 \%$ polymorphonuclear cells, $13 \%$ lymphocytes, $5 \%$ monocytes, $5 \%$ myelocytes, $2 \%$ blast cells and $1 \%$ erythroblasts; red cells abnormalities consisted of anisocytosis, poikilocytosis, polychromasia, macrocytosis, tear drop cells. Bone marrow aspirate was hypocellular with $6 \%$ morphologically unclassifiable blasts, $3 \%$ myeloblasts, $1 \%$ premyelocytes, ${ }^{5} 11 \%$ myelocytes, $22 \%$ band forms and polymorphonuclear cells, and $55 \%$ erythroblasts; megakaryocytes were present. The Prussian blue reaction showed $39 \%$ sideroblasts types I and II and 3\% ringed sideroblasts. Bone marrow biopsy was hypercellular with increased reticulin network (see results). Haemoglobin electrophoresis showed $4.5 \% \mathrm{HbF}$. Serum $B_{12}$ and folate concentrations were normal. Leucocytes alkaline phosphatase score was 174 (normal 20-80). The patient received transfusions for anaemia. A two months treatment with androgens was ineffective. Papular skin lesions were noted in March 1981; a skin biopsy revealed necrotising vasculitis; these lesions regressed spontaneously. In December 1981, the blood count revealed WBC $1.4 \times 10^{9} / 1$ with $64 \%$ polymorphonuclear cells and band forms, $19 \%$ lymphocytes, $7 \%$ monocytes, $10 \%$ myelocytes. A second bone marrow biopsy showed the same abnormalities (see results) of the megakaryocytes as observed in the first marrow 
biopsy; the number of blast cells had not increased. In August 1982, the blood count showed WBC 1.6 $\times 10^{\%} / 1$ with $75 \%$ polymorphonuclear cells and no blast cells, $\mathrm{Hb} 6.6 \mathrm{~g} / \mathrm{dl}$, platelets $5 \times 10^{9} / 1$. The patient died of intracerebral haemorrhage the same month.

\section{Cytological and histological studies}

Blood and bone marrow smears, were stained by the Romanovsky method. ${ }^{78}$ Results of the Prussian blue reaction were interpreted according to Dacie and Mollin. 9 Bone marrow biopsy was fixed in Bouin's fluid, embedded in paraplast and stained with haematoxylin and eosin. Gomori's silver impregnation method was used to demonstrate reticulin network.

Ultrastructural cytochemistry and morphology Peroxidase activity was studied at the time of initial diagnosis by two separate methods.

\section{Method 1}

Fixation in tannic acid-aldehyde mixture ${ }^{10}$ followed by incubation in 3-3'-diaminobenzidine (DAB) medium as previously described. ${ }^{11}$

\section{Method 2 (isolated cells)}

Platelet-rich plasma obtained after centrifugation of whole blood was incubated before fixation in DAB medium. ${ }^{12}$ The medium contained $20 \mathrm{mg}$ of DAB and $0.01 \mathrm{ml}$ of $3 \% \mathrm{H}_{2} \mathrm{O}_{2}$ in $10 \mathrm{ml}$ Tris Ringer $(30$ mg Tris, $10 \mathrm{ml}$ standard Ringer's solution). The $\mathrm{pH}$ was adjusted to $7 \cdot 3$ with $0 \cdot 01 \mathrm{~N} \mathrm{HCl}$. Incubation was carried out in a dark room at $20^{\circ} \mathrm{C}$ for $1 \mathrm{~h}$ after which the cells were rinsed in Tris Ringer and fixed for $30 \mathrm{~min}$ in $1.25 \%$ glutaraldehyde in $0.1 \mathrm{M}$ phosphate. Cytochemical controls for these methods have been previously described. ${ }^{13}$

All the preparations were post fixed for $30 \mathrm{~min}$ in $1 \%$ osmium tetroxide in phosphate buffer, then dehydrated in ethanol and embedded in Epon. Sections cut with a glass knife on Reichert microtome were examined, either unstained or lightly stained with lead citrate, under a Zeiss EM9 and a Philips EM 300 electron microscope.

Methods 1 and 2 were used to study circulating platelets and method 1 was used for the bone marrow.

In addition, the morphology of platelets was studied after fixation by $1.25 \%$ glutaraldehyde in phosphate buffer followed by post-fixation in osmium tetroxide.

\section{Results}

LIGHT MICROSCOPIC STUDY

The marrow smears were hypocellular with numer- ous naked nuclei and basophilic material indicating myelofibrosis. Megakaryocytes were abnormal, often with multiple small nuclei surrounded by an abundant cytoplasm; micromegakaryocytes were also observed. The dyserythropoiesis manifested as vacuolation of the cytoplasm, basophilic stippling, karyorrhexis, bi-or multinuclearity. The granulocytic series showed reduced number of stainable granules; blast cells had a regular outline, a slightly condensed chromatin pattern, one or two prominent nucleoli and an agranular moderately basophilic cytoplasm.

The bone marrow sections were hypercellular with persistence of rare adipocytes. Megakaryocytes were numerous; most of them were small with one or more small nuclei as noticed on smears; these nuclei were round or oval, with a stippled chromatin; the cytoplasm was moderately abundant and eosinophilic; micromegakaryocytes were also observed. Erythroblasts were numerous, at all stages of maturation. The granulocytic series was hypoplastic with presence of some mature forms; there was an excess of blast cells having a round nucleus, often a nucleolus and a moderately abundant cytoplasm; the exact origin of these blast cells was difficult to determine. The reticulin network was extremely dense.

\section{ULTRASTRUCTURAL (EM) MORPHOLOGY AND CYTOCHEMISTRY \\ Bone marrow}

Ultrathin sections revealed a hypercellular marrow. The erythroblasts showed a strong positivity as a result of the pseudoperoxidase activity of the haemoglobin. Some blast cells without granules showed a peroxidase activity present in the perinuclear space and in the endoplasmic reticulum, indicating a non-lymphoid origin. The megakaryocytes had one, two or apparently multiple small nuclei with a rather fine non-condensed chromatin and one or two small nucleoli (Fig. 1). The cytoplasm was often abundant, even in cells with a single small nucleus, contained a variable number of alpha granules, and sometimes a poorly developed demarcation membrane system. The platelet peroxidase activity was highly variable, detectable either in the perinuclear space and in the endoplasmic reticulum or only in the former with a variable intensity; at least half the megakaryocytes were completely devoid of platelet peroxidase activity.

\section{Peripheral blood}

The size and the morphology of the platelets were highly variable. Some giant platelets contained $\stackrel{\infty}{+}$ hypertrophied dense tubular system and were 0 devoid of glycogen, while a majority of small 


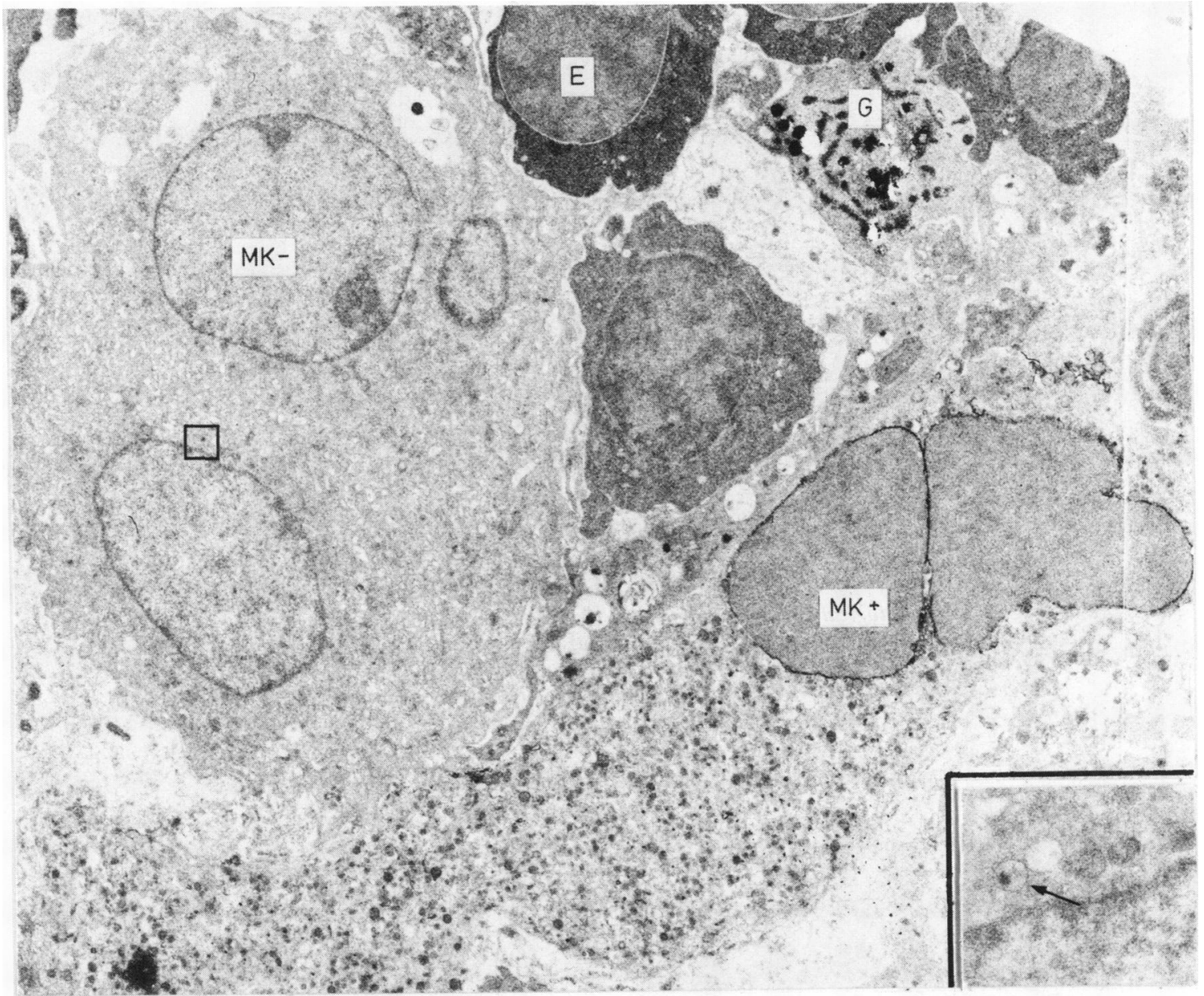

Fig. 1 Two neighbouring megakaryocytes, one platelet peroxidase positive $(M K+)$ and the other platelet peroxidase negative $(M K-)$. Note the positivity of erythroblast $(E)$ and of endoplasmic reticulum and the granules of a granulocytic precursor $(G) \times 6000$. Inset: magnified view of $\alpha$ granule (arrow) in the platelet peroxidase negative megakaryocyte.

platelets exhibited a normal aspect of organelles and clumps of glycogen particles (Fig. 2).

With the method 2 employed to reveal platelet peroxidase, some of the platelets were highly positive, whereas others were without platelet peroxidase. In the latter the absence of penetration of DAB could be excluded since the mitochondria were normally reactive. The platelet peroxidase negative platelets contained glycogen while platelet peroxidase positive platelets, which exhibited little or increased dense tubular system, did not show glycogen (Fig. 3).

This deficiency in platelet peroxidase could also be demonstrated by method 1 (Fig. 4).

\section{Discussion}

Morphological abnormalities of megakaryocytes and platelets are well described in myelodysplastic syndromes: small hypolobulated megakaryocytes, micromegakaryocytes, abnormalities of distribution of demarcation membranes, presence of giant platelets with sometimes abnormal giant granules. ${ }^{614}$ The detection of a peroxidase activity which is specific for the megakaryocytes ${ }^{15}$ allows the recognition of abnormal megakaryocytes and of morphologically unclassifiable blasts which cannot be identified by other techniques as early megakaryoblasts. This technique had been applied 


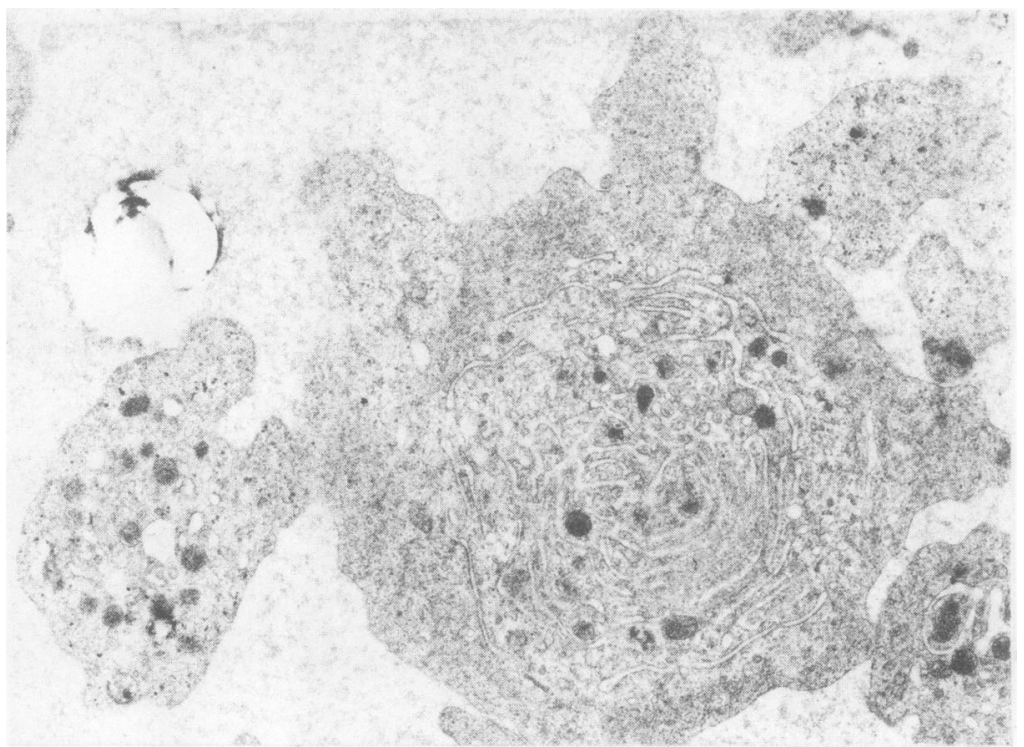

Fig. 2 Morphological features of peripheral blood platelets. The heterogeneity of the size and ultrastructure of platelets are evident; one is large, contains hypertrophied dense tubular system, small number of granules and has no glycogen whereas the other is small, has normal organelles including glycogen. $\times 14400$.
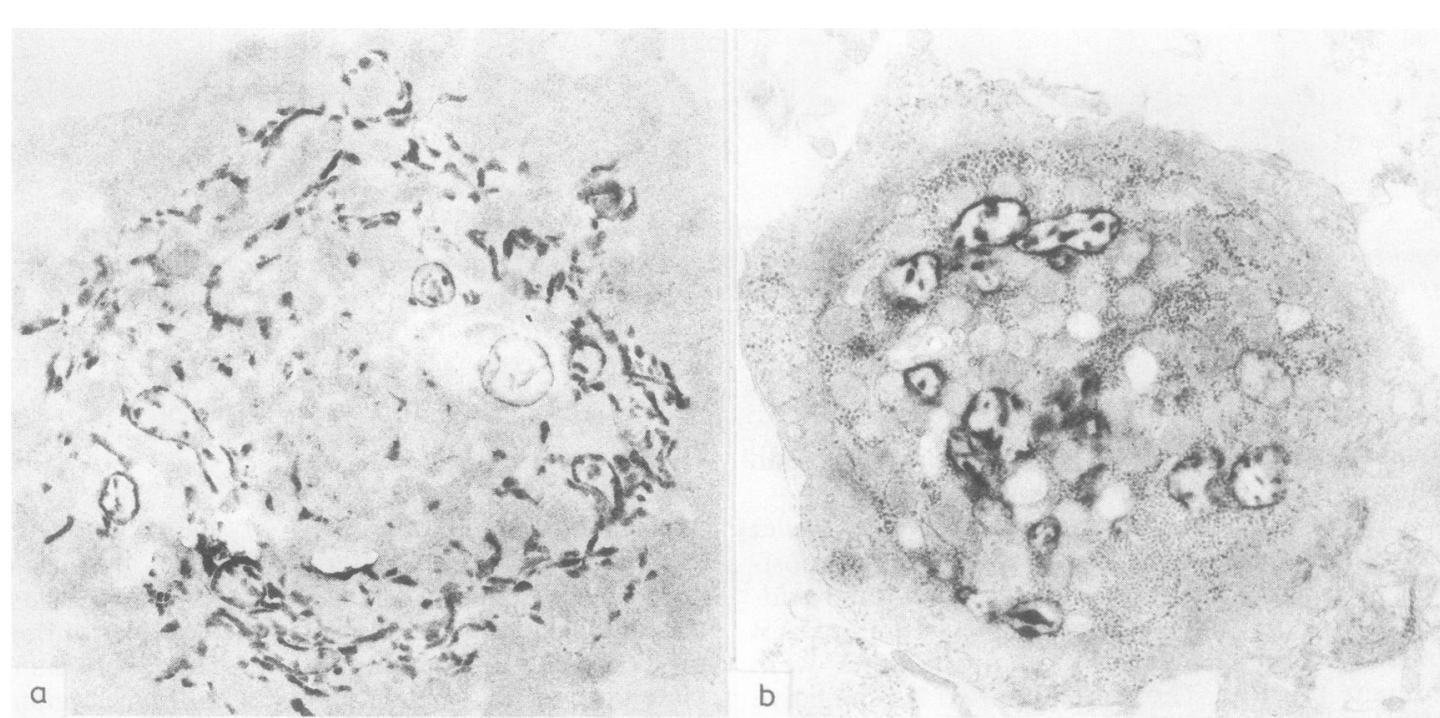

Fig. 3 Platelets incubated in the substrate without prefixation.

$a=$ platelet showing highly developed dense tubular system which exhibits platelet peroxidase reactivity. Note the presence of a membrane complex, the paucity in granules and the absence of glycogen. $\times 20900$;

$b=$ in the same sample, platelet with numerous granules, high content in glycogen but without platelet peroxidase in the dense tubular system. The DAB has normally penetrated into the cell since mitochondria exhibit, as in normal platelets, a peroxidatic activity. $\times 17800$. 


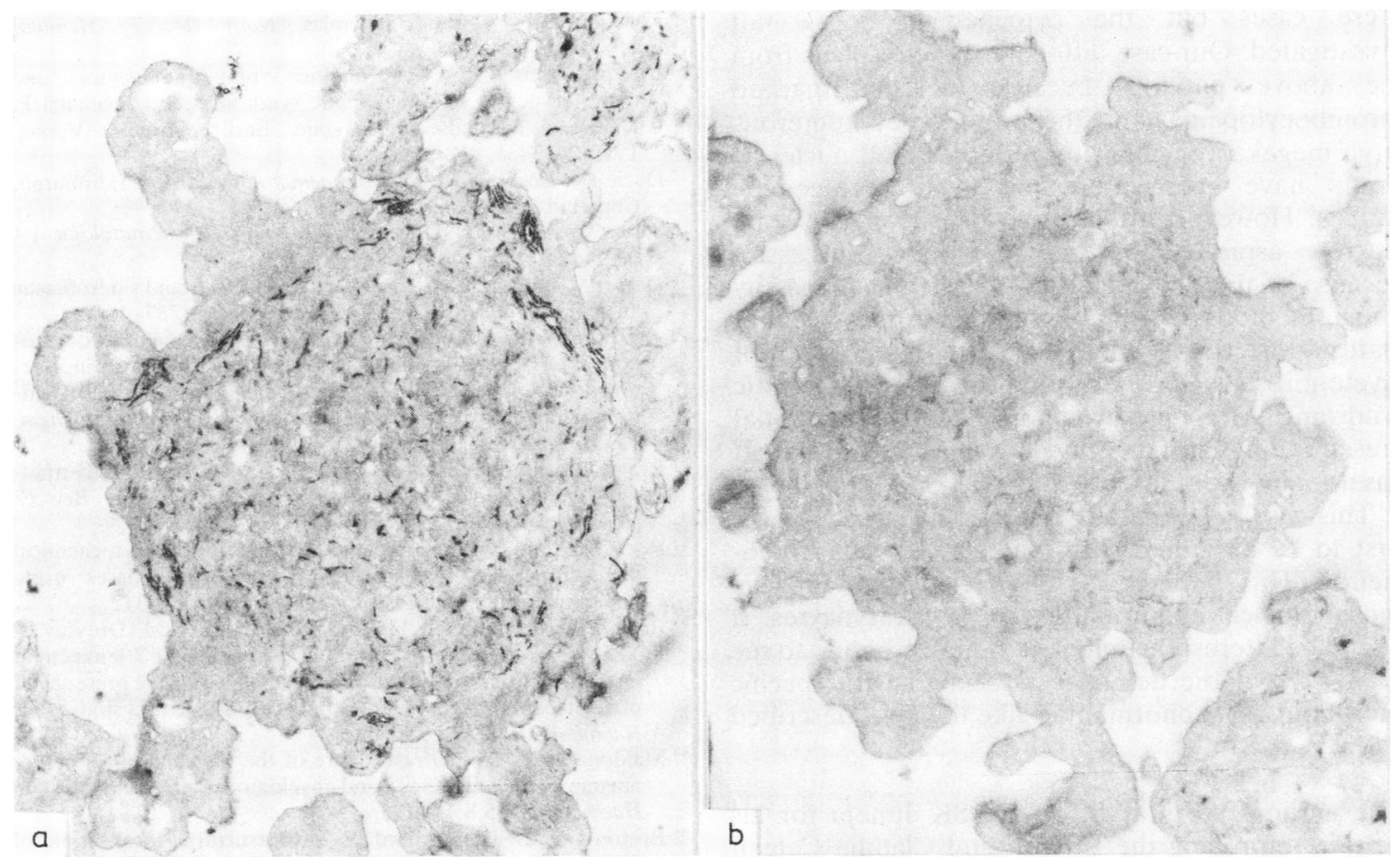

Fig. 4 Method 1. Giant platelets with (a) and without (b) platelet peroxidase activity. $\times 10000$.

routinely for several years by one of the authors (JBG) in cases of myelodysplastic syndromes, acute leukaemias and blast crisis of chronic granulocytic leukaemia. Until now this platelet peroxidase activity has always been demonstrated in megakaryocytic cells even in normal and leukaemic promegakaryoblasts without other evidence of megakaryocytic differentiation-for example, granules or demarcation membranes. ${ }^{16}$

The present case represents the first report of a partial platelet peroxidase deficiency. This absence of platelet peroxidase in some platelets and megakaryocytes is not an artefact for the two following reasons: firstly, in the bone marrow, platelet peroxidase positive and platelet peroxidase negative megakaryocytes coexist in the zones in which the substrate penetrates as indicated by the high reactivity of erythroblasts and granulocytes. The absence of platelet peroxidase in megakaryocytes could be observed in bone marrow as a result of an artefact because of lack of penetration of DAB. In this latter situation the neighbouring granulocytes would exhibit a weak or absent myeloperoxidase activity and red cells and maturing erythroblasts would not show the high density due to the oxidation of DAB. Secondly, the partial platelet peroxidase deficiency was also observed in the peripheral blood by the two methods employed. In our experience, platelets which have been incubated in DAB without fixation are never platelet peroxidase negative. The proof that this absence of reaction in the present case is not the result of a defective penetration of the substrate into the cytoplasm, is that mitochondria exhibited normal peroxidase activity due to cytochrome.

This true platelet peroxidase deficiency in a subpopulation of platelets seems to be the consequence of an absence or defective synthesis of the enzyme by a fraction of megakaryocytes, but it is not possible to rule out that the enzyme is present in an inactive state. The platelet peroxidase is involved in the prostaglandin synthesis which is essential for the normal function of platelets. ${ }^{17}$ Thus, the platelets without platelet peroxidase probably have defective functions. However these functions could not be tested in the present case because of the inability to separate the two populations of platelets and also because of the low platelet count.

Among patients with myelodysplastic syndromes, a group with a distinct karyotypic anomaly, the 5qchromosome has been described recently. ${ }^{18}$ One of the striking features of this group was the presence of megakaryocytes with small round non-lobulated nuclei and giant platelets. Ultrastructural studies of megakaryocytes and platelets were carried out in 
these cases but the cytochemistry was not investigated. Our case differs in some respects from the above patients because of the marked thrombocytopenia, and the presence of numerous large megakaryocytes with multiple small nuclei. It would have been interesting to do cytogenetic studies. However, attempts to do so on the bone marrow aspirate and biopsy were unsuccessful because of the unavailability of cells in mitoses by direct method or after culture. Another peculiar feature of this patient was the presence of myelofibrosis associated with the myelodysplastic syndrome. Although such finding is rare at the initial presentation of primary myelodysplastic syndrome it has already been described. ${ }^{19}$

This case is particularly interesting since it is the first to be described with platelet peroxidase deficiency. This finding was associated with unusual morphological abnormalities of megakaryocytes. It would be interesting to look at other cases and to see if this enzymatic defect is associated with specific morphological abnormalities like the ones described above.

The authors are grateful to Dr SK Juneja for his help in improving the English and Claudie Cateau for secretarial assistance.

\section{References}

' Dreyfus B. Preleukemic states. Definition and classification. Refractory anemia with excess of myeloblasts in the bone marrow. Blood Cells 1976;2:33-55.

${ }^{2}$ Pierre R, Sultan C, Vardiman J. The myelodysplastic syndromes. In: The education program of the American Society of Hematology. Washington, 1982:1-5.

${ }^{3}$ Boivin P. Red blood cell enzyme abnormalities in dyserythropoietic anaemia. In: Lewis SM, Verweilghen RL, eds. Dyserythropoiesis. London: Academic Press, 1977:221-46.

4 Dreyfus B, Sultan C, Rochant H. Anomalies of blood group antigens and erythrocytes enzymes in two types of chronic refractory anaemia. Br J Haematol 1969;16:303-12.
${ }^{5}$ Lewis SM. Dysplastic anaemias. Nouv Rev Fr Hematol 으 1972;12:375-83.

- Breton-Gorius J. Abnormalities of granulocytes and $\overrightarrow{\overline{\vec{C}}}$ megakaryocytes in preleukemic syndromes. In: Schmalzl F, Hellriegel KP, eds. Preleukemia. Berlin: Springer Verlag, 1979:24-34.

7 Dacie JV, Lewis SM. Practical haematology 5th ed. Edinburgh: Churchill Livingstone, 1975.

- Sultan C, Priolet G, Beuzard Y. Techniques en hématologie 1st ed. Paris: Flammarion, 1978

- Dacie JV, Mollin DL. Siderocytes, sideroblasts and sideroblastic anaemia. Acta Med Scand (suppl) 1966;445:237-48.

${ }^{10}$ Anderson WA, Trantalis J, Kang YH. Ultrastructural localization of endogenous mammary gland peroxidase during lac- $\vec{\omega}$ togenesis in the rat. Results after tannic acidformaldehyde-glutaraldehyde fixation. $J$ Histochem Cytochem $\frac{?}{0}$ 1975;23:295-302.

" Breton-Gorius J, Guichard J. Ameliorations techniques permettant de révéler la peroxydase plaquettaire. Nouv $\operatorname{Rev}$ Fr $\vec{\longrightarrow}$ Hematol 1976;16:381-90.

${ }_{12}$ Roels F, Wisse E, De Prest B, Van Der Meulen J. Cytochemical $\vec{N}$ discrimination between catalases and peroxidases using $\mathrm{N}$ diaminobenzidine. Histochemistry 1975;41:281-312.

${ }^{13}$ Breton-Gorius J, Reyes F, Vernant JP, Tulliez M, Dreyfus B. 근 The blast crisis of chronic granulocytic leukaemia: megakaryoblastic nature of cells as revealed by the presence of $Z$ platelet-peroxidase. A cytochemical ultrastructural study. $\mathrm{Br} J$ Haematol 1978;39:295-303.

${ }^{14}$ Maldonado JE. The ultrastructure of the platelets in refractory anemia ("preleukemia") and myelomonocytic leukemia. Ser $\stackrel{\mathbb{Q}}{-}$ Haematol 1975;8:101-25.

15 Breton-Gorius J, Guichard J. Ultrastructural localisation $\mathcal{Q}_{\mathrm{f}} \overrightarrow{\mathrm{\varphi}}$ peroxidase activity in human platelets and megakaryocyt. Am J Pathol 1972;66:277-86.

${ }^{16}$ Breton-Gorius J, Reyes F, Duhamel G, Najman A, Gorin Megakaryoblastic acute leukemia: identification by the ultrastructural demonstration of platelet peroxidase. Blood $\bar{\partial}$ 1978;51:45-60.

17 Gerrard JM, White JG, Rao GHR, Townsend D. Localization of platelet prostaglandin production in the platelet dense tubular@ system. Am J Pathol 1976;83:283-94.

18 Sokal G, Michaux JL, Van Den Bergue H et al. A new음 hematological syndrome with distinct karyotype: the 5qchromosome. Blood 1975;46:519-33.

19 Sultan C, Sigaux F, Imbert M, Reyes F. Acute myelodysplastic with myelofibrosis: a report of eight cases. $\mathrm{Br} \mathrm{J}$ Haematol 1981;49:11-16.

Requests for reprints to: Dr M Imbert, Service Central d'Hématologie-Immunologie, Hôpital Henri Mondor, 94010 Creteil, France. 